\title{
Local modulated electro-hyperthermia in combination with traditional Chinese medicine vs. intraperitoneal chemoinfusion for the treatment of peritoneal carcinomatosis with malignant ascites: A phase II randomized trial
}

\author{
CLIFFORD L.K. PANG ${ }^{1}$, XINTING ZHANG ${ }^{1}$, ZHEN WANG $^{2}$, JUNWEN OU $^{3}$, YIMIN LU ${ }^{4}$, \\ PENGFEI CHEN $^{5}$, CHANGLIN ZHAO $^{5}$, XIAOPU WANG ${ }^{5}$, HONGYU ZHANG $^{5}$ and SERGEY V. ROUSSAKOW ${ }^{6}$ \\ ${ }^{1}$ International Natural Medicine Center; ${ }^{2}$ Scientific Research Center; ${ }^{3}$ Nutrition and Preventive Medicine Center; \\ ${ }^{4}$ Hyperthermia Center; ${ }^{5}$ Nontoxic Integrative Cancer Treatment Center, Clifford Hospital, Guangzhou, \\ Guangdong 511495, P.R. China; ${ }^{6}$ Galenic Research Institute for Non-Specific Pathology, 127051 Moscow, Russia
}

Received June 16, 2016; Accepted December 16, 2016

DOI: $10.3892 / \mathrm{mco} .2017 .1221$

\begin{abstract}
The purpose of this study was to develop a safe and non-toxic alternative to the conventional conservative treatment of peritoneal carcinomatosis with malignant ascites (PCMA) by investigating the efficacy and safety of local modulated electro-hyperthermia (mEHT) combined with the traditional Chinese medicine (TCM) 'Shi Pi' herbal decoction, compared with standard intraperitoneal chemoinfusion (IPCI). A randomized, controlled, single-center, open-label clinical trial (phase II) with two parallel groups (allocation ratio, 1:1) was conducted to investigate the efficacy and safety of mEHT+TCM (study group, SG) vs. standard IPCI (control group, CG) in patients with PCMA by intention-to-treat analysis. A total of 260 patients with PCMA were randomly allocated into the two groups (130/130); mEHT was applied
\end{abstract}

Correspondence to: Dr Sergey V. Roussakow, Galenic Research Institute for Non-Specific Pathology, Office 36, Building 1, 9 M. Sukharevskiy Street, 127051 Moscow, Russia

E-mail: roussakow@gmail.com

Abbreviations: AER, adverse event rate; CG, control group; CR, complete remission; CRPE, cytoreductive peritonectomy; CTCAE, Common Terminology Criteria for Adverse Events; HIPEC, hyperthermic intraperitoneal chemoperfusion; HT, hyperthermia; IPCI, intraperitoneal chemoinfusion; IPCP, intraperitoneal chemoperfusion; ITT, intention-to-treat analysis; KPS IR, Karnofsky performance score improvement rate; MA, malignant ascites; mEHT, modulated electro-hyperthermia; NC, no change; ORR, objective response rate; PC, peritoneal carcinomatosis; PCMA, peritoneal carcinomatosis with malignant ascites; PR, partial remission; QoL, quality of life; RCT, randomized controlled trial; SG, study group; VAS, visual analog scale

Key words: modulated electro-hyperthermia, traditional Chinese medicine, malignant ascites for 60 min per session every second day for 4 weeks, for a total of 14 sessions. The TCM decoction was administered orally, at $400 \mathrm{ml}$ daily. In CG, occlusive IPCI with cisplatin (30-60 mg) and fluorouracil (500-600 mg/m²) was applied twice, biweekly. The objective response rate (ORR), quality of life (QoL) and adverse event rate (AER) in the two groups were evaluated 1 month after treatment, analyzed and compared. The present study is registered on ClinicalTrials.gov (NCT02638051). No case was lost or excluded (0/260). The ORR in SG was $77.69 \%$ $(101 / 130)$ vs. $63.85 \%$ (73/130) in CG $(\mathrm{P}<0.05)$. The $\mathrm{QoL}$ in SG was $49.23 \%$ vs. $32.3 \%$ in $\mathrm{CG}(\mathrm{P}<0.05)$. The AER in $\mathrm{SG}$ was $2.3 \%(3 / 130)$ vs. $12.3 \%(16 / 130)$ in $\mathrm{CG}(\mathrm{P}<0.05)$. All the adverse events were grade I. In conclusion, the combination of mEHT with TCM achieves better control of PCMA compared with standard IPCI, with less toxicity. Both components of the combination are non-toxic treatments easily tolerated by patients. Thus, this combined treatment may be preferred due to the better benefit-harm balance.

\section{Introduction}

Malignant ascites (MA) is a common clinical manifestation of advanced-stage abdominal cancer. Peritoneal carcinomatosis (PC) is the cause of $>50 \%$ of the cases of MA (PCMA). In $>50 \%$ of PC patients, MA is the first clinical symptom of the disease (1). The appearance of MA indicates an unfavorable prognosis, with an expected median survival time of $\sim 20$ weeks from the time of diagnosis (2). Reduction of MA may improve the quality of life $(\mathrm{QoL})$ of the patients, create conditions for further anticancer treatments and prolong survival.

As the existing conservative treatment of PCMA is mainly based on different forms of chemotherapy with its inherent toxicity, there is a strong demand for a safe and non-toxic method for the treatment of this condition. The objective of the present trial was to develop such an alternative for conventional chemotherapy-based treatments of PCMA.

In addition to conventional cancer treatments, hyperthermia (HT) is the most widely investigated complementary 
cancer treatment modality for the conservative treatment of PCMA in combination with chemotherapy (Table I). However, although temperature-based HT has a long history of application in oncology, its efficacy and safety remain unproven $(3,4)$ and it is currently considered as experimental treatment (5).

The new technology of modulated electro-HT (mEHT; Oncothermia ${ }^{\mathrm{TM}}$ ) is drawing increasing attention due to its minimal side effects and synergy with other treatments (6). mEHT is based on the nano-thermal but not temperature-dependent effects of electromagnetic fields and special modulation (7), whose effect may exceed the effect of the overall heating (macroscopic temperature elevation) by 3-4-fold (8). mEHT does not require hyperthermia-range temperature and may be performed safely, without invasive thermal control. Unlike conventional hyperthermia, mEHT is also effective as monotherapy $(7,9)$. Our previous phase II randomized trial on the combination of mEHT with traditional Chinese medicine (TCM) in colorectal cancer (9) suggested a synergetic effect of mEHT and TCM and was found to be sufficiently beneficial in PCMA patients for the present trial to be initiated.

TCM has a long history of application in patients with advanced cancer as symptomatic treatment and enhancer of the general resistance of the organism (10). TCM is holistic medicine, which treats the body as a whole combined with lifestyle and environmental factors, describing it in terms of 'vital energy', also referred to as 'Qi'.

In terms of TCM, MA belongs to the category of 'Gu Zhang' (11), which means tympanites or distension of the abdomen caused by the accumulation of gas or fluid (12). The pathogenesis of $\mathrm{Gu}$ Zhang is connected with illness of three organs, namely the liver, spleen and kidney, which leads to stasis of Qi, blood and water in the abdomen, leading to abdominal distention and, finally, the formation of Gu Zhang. The application of TCM effectively relieves the symptoms and improves the QoL of patients with MA (12) by clearing heat and removing dampness, purging water, promoting blood flow and relieving blood stasis, promoting the circulation of Qi and dissipating dampness, invigorating the spleen and kidney, and dissipating warmth resolving watery dampness (13).

The number of clinical trials on TCM as co-treatment for PCMA (Table II) using different TCM treatments is limited (14-17). The Shi Pi decoction is considered to be the optimal treatment for $\mathrm{Gu}$ Zhang. This decoction was first described in Ji Sheng Fang (Life-saving Prescriptions) (18). This may warm Yang, invigorate the spleen and promote Qi circulation to induce diuresis. The main effect of the Shi Pi decoction is treatment of the Foot-Taiyin meridian.

The optimal or standard treatment is considered as the control treatment in randomized studies. In clinical practice, diuretics, abdominal paracentesis and local injection of biological agents and chemotherapeutic drugs are commonly used to treat PCMA (19). The standard treatment for PCMA is currently intraperitoneal chemotherapy applied as chemoinfusion (IPCI) or chemoperfusion (IPCP). IPCP is usually performed following cytoreductive peritonectomy (CRPE), whereas IPCI is mainly a stand-alone conservative procedure. Although IPCP/CRPE appears to be superior to IPCI, it is a difficult, high-risk and costly procedure. Furthermore, there are not sufficient data to conclude on IPCP feasibility in this combination (20). Hyperthermic intraperitoneal chemoperfusion (HIPEC) appears to be the most commonly used IPCP method providing better survival times, although it is currently not recommended as a standard-of-care option due to the controversy surrounding its use (21). In addition, there is no definitive proof supporting the advantage of HIPEC over normothermic IPCP, and accumulating preclinical data suggest that HIPEC has no advantage over IPCP (22-24). Hyperthermia has already been proven to be of no value in isolated limb chemoperfusion (25). IPCP is associated with the inherent toxicity of chemotherapy and additional toxicity due to damage of the peritoneum (26) and septic shock (27). In China, IPCI with cisplatin and fluorouracil is a widely used standard treatment for PCMA $(28,29)$ (Table I).

\section{Patients and methods}

Study design. The present study was a randomized, controlled, single-center, open-label clinical trial (phase II) with two parallel groups (allocation ratio, 1:1), which was conducted to investigate the efficacy and safety of mEHT in combination with TCM (study group, SG) vs. standard IPCI (control group, $\mathrm{CG})$ in PCMA by intention-to-treat analysis.

Inclusion and exclusion criteria. Patients who were hospitalized at the Department of Oncology of Clifford Hospital (Guangzhou, China) were recruited based on the following inclusion criteria: i) Pathologically confirmed PC with malignant ascites; ii) Karnofsky performance status (KPS) score (30) $\geq 60 \%$; iii) normal function of bone marrow; iv) predicted survival time $>1$ month; and v) written informed consent. The exclusion criteria were as follows: i) Surgery within 3 weeks or incomplete recovery of postoperative sutures; ii) active bleeding or vascular occlusion in the mEHT treatment area; iii) emotional instability; iv) difficulty in placing the patient into the mEHT machine; v) metallic implants or replacements in the treatment area; vi) implanted electronic devices; vii) missing or damaged heat-sense nerves or other field-sensitive issues in the treatment area; and viii) very low white blood cell count $\left(<1.5 \times 10^{9} / 1\right)$, agranulocytosis $\left(<0.5 \times 10^{9} / 1\right)$ or severe anemia. Disease staging was performed according to the NCCN staging criteria (31).

\section{Interventions}

Local mEHT. For local mEHT, the EHY-2000 local oncothermia device was applied (Oncotherm $\mathrm{GmbH}$, Troisdorf, Germany). Treatment was performed in the supine position with a $30-\mathrm{cm}$ diameter electrode, 60 min per session. Step-up heating was applied, with a power increasing from 100 to $150 \mathrm{~W}$ over 5-15 min according to the heat tolerance of the patient. mEHT was applied every second day within 4 weeks, for a total of 14 sessions.

TCM. The composition of the Shi Pi base decoction was as follows: i) Atractylodes macrocephala koidz (15 g); ii) Cortex magnoliae officinalis (10 g); iii) Chaenomeles sinensis (Thouin) Koehne (9 g); iv) Radix Aucklandiae (6 g); v) Fructus Tsaoko (5 g); vi) Areca catechu L. (9 g); vii) Poria cocos (Schw.) Wolf (15 g); viii) Rhizoma zingiberis (9 g); ix) Radix Aconiti Lateralis Preparata (6 g); x) Radix Glycyrrhizae Preparata 
(9 g); xi) Zingiber officinale Roscoe (3 slices); and xii) Fructus jujubae (3 pcs.).

The composition was adjusted according to the diagnostic criteria of treatment determination based on pathogenesis obtained through differentiation of symptoms and signs of TCM (32) as follows:

Syndrome of accumulated dampness-heat: Distension and hardness of abdomen, tympanites and pain in the gastric cavity and abdomen, feverish dysphoria, bitter taste in the mouth, feeling of thirst but unwillingness to drink, occasionally yellow discoloration of the face, eyes and skin of the body, oliguria with yellow urine, constipation, red tongue with greasy yellow coating, and stringy, rapid pulse.

Adjustment: Exclusion of Rhizoma zingiberis and Radix Aconiti Lateralis; addition of Radix Stephaniae Tetrandrae (15 g), Lepidium apetalum Willd. (12 g), Fructus Gardeniae (15 g) and Herba Artemisiae Scopariae (20 g).

Qi-stagnancy and blood stasis type: Distended abdomen, varicose veins on the abdominal wall, prickling flank pain, unpressable pain, swarthy grey discoloration of the face, violet discoloration of the lips, vascular nevus on the cheeks and chest of snake-like shape or striped, red marks on the palms, feeling of thirst but unwillingness to drink, black stool, purple red tongue with yellow coating and thready, irregular pulse.

Adjustment. Exclusion of Atractylodes macrocephala koidz. and Radix Aconiti Lateralis; addition of Rhizoma cyperi (12 g), Semen persicae (15 g), Cortex Moutan (12 g) and Radix paeoniae rubra (12 g).

Asthenia of the spleen and kidney type. Distended abdomen, epigastric distension and depression, anorexia, loose stool, tiredness, aversion to cold, edema of lower limbs, paleness of the face, pale tongue with thin white coating and deep, thready, weak pulse.

Adjustment: Addition of Polyporus umbellatus (Pers) Fr. $(15 \mathrm{~g})$ and Radix Aconiti Lateralis ( $\leq 12 \mathrm{~g})$.

A total of $400 \mathrm{ml}$ of decoction were prepared from each dose of the herbs. The decoction was administered orally for 4 weeks, twice a day, $200 \mathrm{ml}$ each time, $30 \mathrm{~min}$ after breakfast and supper.

IPCI. Abdominal paracentesis was performed by catheterization following closed drainage of the ascites with only a small amount of residual fluid, and was followed by infusion of cisplatin (30-60 mg) and fluorouracil $\left(500-600 \mathrm{mg} / \mathrm{m}^{2}\right.$ body surface); dose reductions were applied depending on the general condition of each patient. Each medication was dissolved in $100 \mathrm{ml}$ of normal saline. Following IPCI, the catheter was occluded. IPCI was performed every 2 weeks during the 4-week treatment course, for a total of two courses.

Outcomes. The primary outcome was objective response rate (ORR); the secondary outcomes were AER and QoL. Tumor response was assessed according to the World Health Organization criteria (33) for evaluation of the therapeutic effect in MA: Complete remission (CR) was defined as complete absorption of the ascites with no obvious regeneration for $>1$ month; partial remission (PR) was defined as $>50 \%$ reduction of the ascites, with obvious relief of the abdominal distention, with maintenance of a less than moderate volume of ascites under ultrasound detection for $>1$ month; and no change (NC) was defined as $<50 \%$ reduction of the ascites, or no obvious reduction of the ascites under ultrasound detection, or even increase of the ascites, with obvious abdominal distention. ORR was calculated as CR + PR.

QoL was assessed by KPS improvement rate (KPS IR) and pain according to the KPS IR criteria (20) as follows: (i) Improvement was defined as a KPS increase of $\geq 10 \%$ after treatment; (ii) worsening was defined as a KPS reduction of $\geq 10 \%$ after treatment; and (iii) $\mathrm{NC}$ was defined as a change in KPS of $<10 \%$.

The pain was assessed using the visual analog scale (VAS) (34) and AER in accordance with the Common Terminology Criteria for Adverse Events (CTCAE) (35).

Sample size and randomization. The sample size was determined as follows: $\mathrm{n}=\left(\mathrm{U}_{\alpha}+\mathrm{U}_{\beta}\right)^{2} \times 2 \mathrm{P} \times\left(1 \_\mathrm{P}\right) /\left(\mathrm{P}_{1} \_\mathrm{P}_{0}\right)^{2}$, where $n$ is the sample size of the $\mathrm{SG}$ (same for $\mathrm{CG}$ ); $\mathrm{U}_{\alpha}$ and $\mathrm{U}_{\beta}$ are the corresponding $U$ values for $\alpha=0.05$ and $\beta=0.01$ according to normal distribution quantile table; $\mathrm{P}_{0}$ and $\mathrm{P}_{1}$ are the expected ORR in CG and SG, respectively, estimated by the previous results of TCM + mEHT and IPCI at Clifford Hospital; $\mathrm{P}$ is the average ORR. $\mathrm{P}_{1}=80 \%, \mathrm{P}_{0}=62 \% ; \mathrm{U}_{\alpha}(0.05)=1.65$, $\mathrm{U}_{\beta}(0.01)=1.28$ and $\mathrm{P}=\left(\mathrm{P}_{1}+\mathrm{P}_{0}\right) / 2=0.71$; therefore $\mathrm{n}=(1.65+1.28)^{2}$ $\mathrm{x} 2 \times 0.71 \times(1-0.71) /(0.8-0.62)^{2}=109$.

As $\sim 15 \%$ of the sample could be lost, 'n' should be $\geq 126$; thus, 130 was defined as the size of the SG.

The patients were randomly distributed into two groups (SG and CG) according to a random number table with an allocation ratio of 1:1. For each patient, the dichotomous decision on the treatment regimen was made by a random allocation sequence and placed into an opaque, sealed, sequentially numbered envelope. When the patient consented to entering the trial, the respective envelope was opened and the appropriate intervention was applied.

There was no blinding, as concealing mEHT treatment from both patients and personnel was not feasible (open-label trial). In order to prevent selection bias and to ensure proper allocation concealment, recruiting, allocation, operation, evaluation of therapeutic effect and data analysis were performed by different individuals. The 'Chinese wall policy' was applied to prevent any communication regarding the trial between different segments of the trial process.

Data collection and management. All the patients were evaluated after each treatment by B-mode ultrasound, clinical and laboratory examinations. The adverse events were evaluated based on objective data and voluntary testimonies of the patients, or through non-leading questions, and were recorded into a 'table of adverse events'.

Data were analyzed by SPSS 19.0 software (IBM Corp, Armonk, NY, USA). Quantitative data were assessed using the $t$-test and multifactor analysis of variance. Data with non-normal distribution were assessed using the rank-sum test. Categorical data were assessed by the Chi-squared test. 


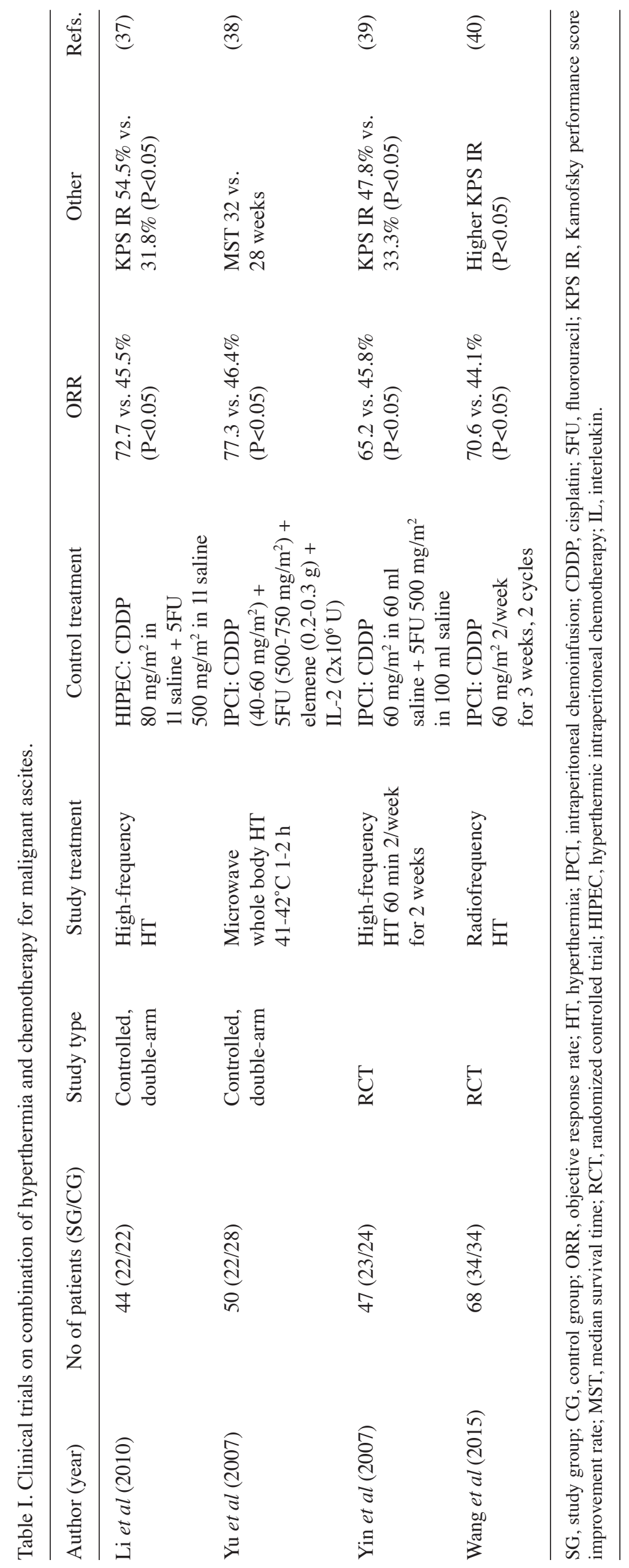




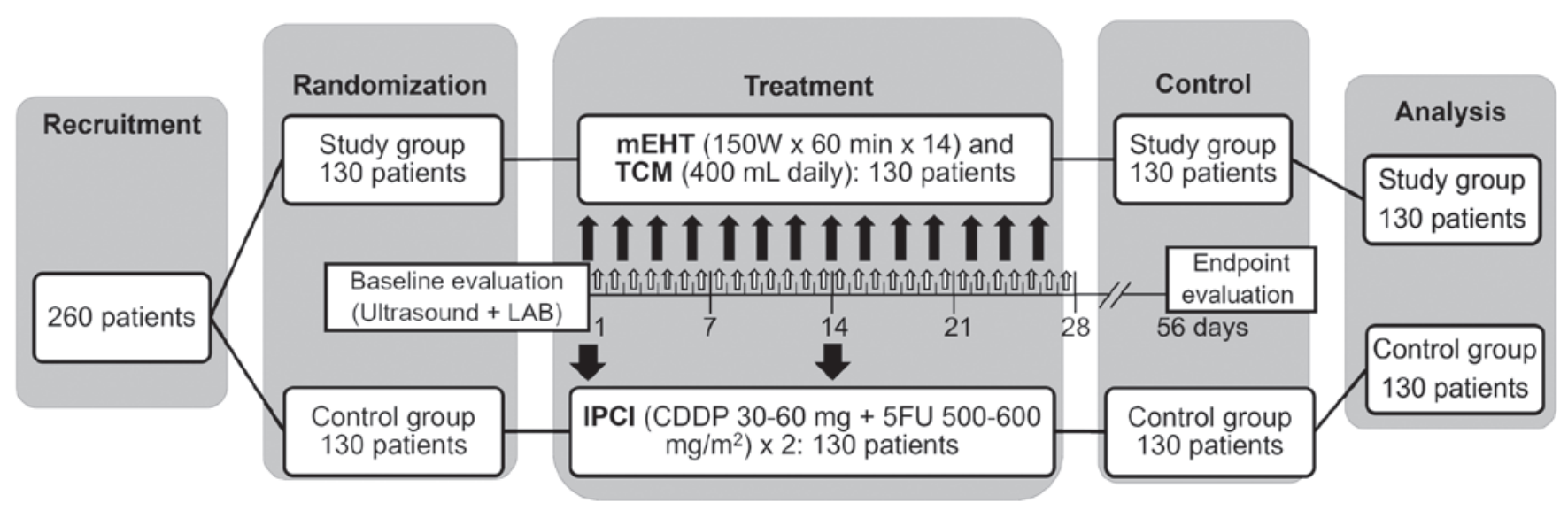

Figure 1. Trial protocol. mEHT, modulated electro-hyperthermia; TCM, traditional Chinese medicine herbal decoction; CDDP, cisplatin; 5FU, 5-fluorouracil; LAB, laboratory investigations. Thin black arrows, mEHT sessions; bold black arrows, IPSI sessions; white arrows, TCM sessions.

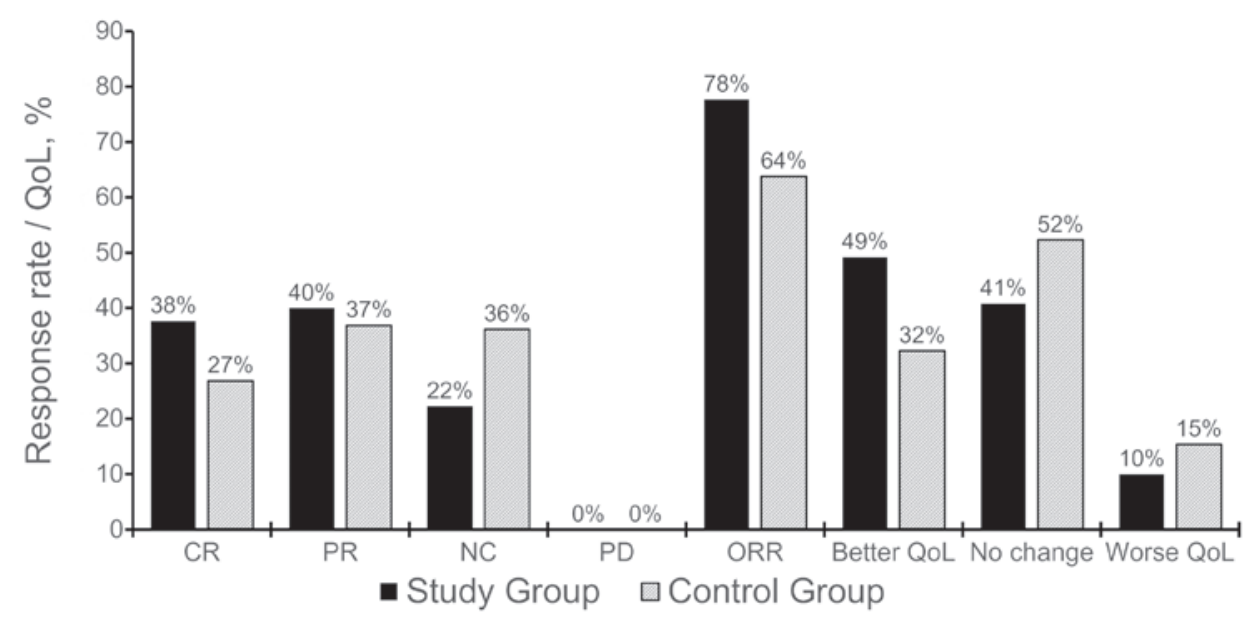

Figure 2. Effect of treatment in terms of objective response rate (ORR) and quality of life (QoL). CR, complete remission; PR, partial remission; NC, no change; $\mathrm{PD}$, progressive disease.

The present study was performed in accordance with the CONSORT 2010 statement (36) and registered on ClinicalTrials.gov (NCT02638051).

\section{Results}

Patients and treatment. Between January 3 and December 20, 2014, 260 patients were recruited at the Clifford Hospital. The patients were randomly allocated into the two groups, with 130 patients assigned to each group (Fig. 1). There was no dropout or exclusion following randomization (0/260). All the patients in the SG (130/130) received the complete prescribed local mEHT + TCM treatment course, whereas all the patients in the CG (130/130) received the complete prescribed ICPI course (Fig. 1) with the following average/median doses: CDDP, $49.63 \pm 10.19 / 50 \mathrm{mg} ; 5 \mathrm{FU}, 48.5 \pm 39.68 / 550 \mathrm{mg} / \mathrm{m}^{2}$. There were no significant differences in symptomatic supportive treatment between the two groups. All the patients (260/260) were recorded and analyzed on an intention-to-treat basis.

Baseline data. In the SG, the age range was $27-73$ years, with a mean \pm standard deviation (SD) of $58.88 \pm 12.43$ years. In the $\mathrm{CG}$, the age range was $24-75$ years, with a mean $\pm \mathrm{SD}$ of
$56.07 \pm 15.38$ years $(\mathrm{P}=0.11$; Table III). The percentage of male patients was 55.4 and $46.9 \%$ in the SG and CG, respectively $(\mathrm{P}=0.17)$. The primary disease was gastric cancer $(16.0$ and $18.5 \%$ in the SG and CG, respectively), colon cancer (26.2 and $28.5 \%)$, rectal cancer (13.8 and $11.5 \%)$, pancreatic cancer (10.0 and 6.2\%), endometrial cancer (6.9 and 3.8\%), ovarian cancer (8.5 and 12.3\%) and hepatic cancer (17.7 and 19.2\%). There was no statistically significant difference in terms of primary disease between the two groups ( $\mathrm{P}=0.7)$. In $\mathrm{SG}$ and $\mathrm{CG}$, the rate of lung metastasis was 17.7 and $15.4 \%$, of liver metastasis 24.6 and $26.9 \%$, of metastasis to the celiac lymph nodes 40.8 and $38.5 \%$ and to the bones 16.9 and $19.2 \%$, respectively. There was no statistically significant difference in the rate and location of metastases between the two groups $(\mathrm{P}=0.89)$. There were no stage I/II patients. Stage III patients comprised 50.8\% of SG and $58.5 \%$ of CG. There were more stage IV patients in SG vs. those in CG (49.2 vs. $41.5 \%$, respectively), although the difference was not statistically significant $(\mathrm{P}=0.21)$. The performance status was similar between the two groups ( $\mathrm{P}=0.76$; Table III).

Outcomes and estimation. In the SG, 101 patients (77.69\%) exhibited an objective response (CR + PR) vs. 73 patients 
$(63.85 \%)$ in the CG; the difference was statistically significant $\left(\chi^{2}=6.02, P=0.005\right)$. SG patients also exhibited a better $\mathrm{CR}$ rate (37.7 vs. $26.9 \%$ ), although the difference was not statistically significant $(\mathrm{P}=0.063)$. The KPS improved in $49.23 \%$ of SG patients vs. $32.3 \%$ of CG patients; the difference was statistically significant $\left(\chi^{2}=7.54, \mathrm{P}<0.05\right.$; Table IV and Fig. 2$)$.

The total AER was $2.3 \%$ (3 cases) in the SG vs. $12.3 \%$ (16 cases) in the CG $(\mathrm{P}<0.05$; Table $\mathrm{V})$. In $\mathrm{SG}$, all the patients exhibited mild abdominal pain due to distention. In CG, 5 patients exhibited abdominal pain, 3 had gastrointestinal reactions, 2 had compromised hepatic or renal function, and 6 patients exhibited bone marrow suppression. All the adverse events were grade 1 and they were relieved spontaneously without special treatment.

\section{Discussion}

In this study, mEHT was applied in combination with an orally administered herbal TCM decoction to treat PCMA. mEHT + TCM treatment was found to achieve better control of PCMA compared with standard IPCI, with lower toxicity: The ORR in SG vs. CG patients was 77.69 vs. $63.85 \%$, respectively $(\mathrm{P}<0.05)$, and the KPS improvement rate was 49.23 vs. $32.3 \%$, respectively $(\mathrm{P}<0.05)$, without any significant adverse reactions. Therefore, this combined treatment may be preferred due to the better balance of benefit and harm.

A comparison of the results of the present study to those of previous HT + TCM studies for PCMA (Tables I-II), suggests that our results were superior (ORR, 77.7 vs. $74.5 \%$ in the SG and 63.8 vs. $48.0 \%$ in the CG, respectively; Fig. 3). It should be noted that three of the five previous studies were non-randomized $(14,37,38)$, whereas the two RCTs $(39,40)$ were small-sized (47 and 68 patients in total, respectively). Randomization and sample increase significantly decrease the selection and informational bias, leading to a significant reduction of the absolute and relative efficacy. Moreover, the study by Chen (14) with the highest ORR included $33 \%$ of patients with malignant pleuritis, which is clinically a more easily manageable condition, and the overall population in our study had more advanced disease (49.2\% of the SG patients had stage IV disease) compared with the other studies.

However, our RCT, which included a larger sample [ $>2$-fold compared with the nearest trial (14)] and was well-randomized, demonstrated a better ORR compared with four of five comparative studies, and a significantly better result in the control arm compared with all the previous trials. The result in the CG is of major significance, as it was on the level of the SG results from other RCTs (64 vs. 65-71\%) and significantly higher compared with their CGs (64 vs. $42-44 \%)(39,40)$. Such a significant superiority in treatment efficacy (higher by $33 \%$ compared with the mean of the previous studies) indicates significantly better treatment control, which is pivotal to the quality of an RCT, as inadequate control is a well-known and widespread cause of bias. Although the relative increase of the ORR in our study was significantly lower compared with that in previous trials (22 vs. $55 \%$, respectively), this is entirely due to the significantly better ORR in the control arm, which emphasizes the quality of the trial.

In our trial, the relief of MA was usually associated with a significant decrease or even disappearance of PC 
Table III. Baseline demographic and clinical characteristics.

\begin{tabular}{|c|c|c|c|c|}
\hline Characteristics & Study group & Control group & $\chi^{2}$ & P-value \\
\hline No. recruited/analyzed & $130 / 130$ & $130 / 130$ & & 0.106 \\
\hline Age years $($ mean $\pm \mathrm{SD})$ & $58.88 \pm 12.43$ & $56.07 \pm 15.38$ & & \\
\hline Gender, n (\%) & & & 1.863 & 0.172 \\
\hline Male & $72(55.4)$ & $61(46.9)$ & & \\
\hline Female & $58(44.6)$ & $69(53.1)$ & & \\
\hline Type of cancer, n (\%) & & & 3.829 & 0.700 \\
\hline Gastric & $22(16.9)$ & $24(18.5)$ & & \\
\hline Colon & $34(26.2)$ & $37(28.5)$ & & \\
\hline Rectal & $18(13.8)$ & $15(11.5)$ & & \\
\hline Pancreatic & $13(10.0)$ & $8(6.2)$ & & \\
\hline Endometrial & $9(6.9)$ & $5(3.8)$ & & \\
\hline Ovarian & $11(8.5)$ & $16(12.3)$ & & \\
\hline Hepatic & $23(17.7)$ & $25(19.2)$ & & \\
\hline Metastases, n (\%) & & & 0.622 & 0.891 \\
\hline Lungs & $23(17.7)$ & $20(15.4)$ & & \\
\hline Liver & $32(24.6)$ & $35(26.9)$ & & \\
\hline Celiac lymph nodes & $53(40.8)$ & $50(38.5)$ & & \\
\hline Bones & $22(16.9)$ & $25(19.2)$ & & \\
\hline Stage, n (\%) & & & 1.552 & 0.213 \\
\hline I & $0(0.0)$ & $0(0.0)$ & & \\
\hline II & $0(0.0)$ & $0(0.0)$ & & \\
\hline III & $66(50.8)$ & $76(58.5)$ & & \\
\hline IV & $64(49.2)$ & $54(41.5)$ & & \\
\hline Karnofsky performance score, n (\%) & & & 1.179 & 0.758 \\
\hline 60 & $26(20.0)$ & $21(16.2)$ & & \\
\hline 70 & $50(38.5)$ & $47(36.2)$ & & \\
\hline 80 & $42(32.3)$ & $48(36.9)$ & & \\
\hline 90 & $12(9.2)$ & $14(10.8)$ & & \\
\hline
\end{tabular}

SD, standard deviation.

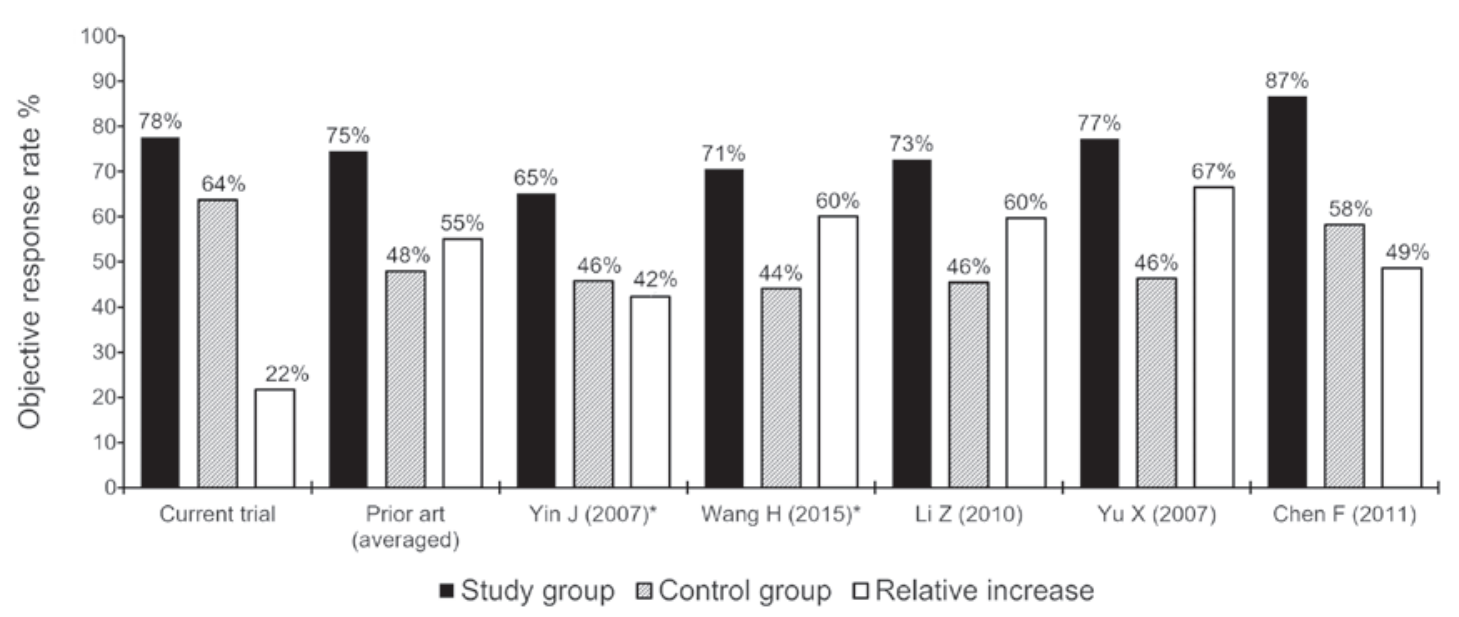

Figure 3. Comparison of the objective response rate between the present study and the previously published literature. *, randomized trials.

manifestations (Fig. 4). In addition, although ascites in a proportion of SG patients was not completely absorbed after
4 weeks of treatment, it was further absorbed to different degrees (even completely absorbed) at the 1-month follow-up 
Table IV. Treatment results.

\begin{tabular}{|c|c|c|c|c|c|c|c|c|c|c|}
\hline \multirow[b]{2}{*}{ Groups } & \multirow{2}{*}{$\begin{array}{l}\text { Total } \\
\text { cases, n }\end{array}$} & \multicolumn{5}{|c|}{ Objective response, n (\%) } & \multicolumn{4}{|c|}{ QoL, n (\%) } \\
\hline & & $\mathrm{CR}$ & PR & $\mathrm{NC}$ & PD & ORR & Better & $\mathrm{NC}$ & Worse & KPS IR, \% \\
\hline Study & 130 & 49 (37.7) & $52(40.0)$ & $29(22.3)$ & $0(0.0)$ & $101(77.7)$ & $64(49.2)$ & $53(40.8)$ & $13(10.0)$ & 49.2 \\
\hline Control & 130 & 35 (26.9) & $48(36.9)$ & $47(36.2)$ & $0(0.0)$ & $83(63.8)$ & $42(32.3)$ & $68(52.3)$ & $20(15.4)$ & 32.3 \\
\hline $\mathrm{P}$-value & \multicolumn{6}{|c|}{$<0.05$} & \multicolumn{4}{|c|}{$<0.05$} \\
\hline
\end{tabular}

CR, complete response; PR, partial response; NC, no change; PD, progressive disease; ORR, objective response rate; QoL, quality of life; KPS IR, Karnofsky performance score improvement rate.

Table V. Adverse event rate.

\begin{tabular}{|c|c|c|c|}
\hline Adverse events & Study group, n (\%) & Control group, n (\%) & P-value \\
\hline Total & $3 / 130(2.31)$ & $16 / 130(12.31)$ & $<0.05$ \\
\hline \multicolumn{4}{|c|}{ Blood and lymphatic system disorders } \\
\hline Bone marrow suppression & $0 / 130(0.00)$ & $6 / 130(4.62)$ & $<0.05$ \\
\hline \multicolumn{4}{|l|}{ Gastrointestinal disorders } \\
\hline Abdominal pain & $3 / 130(2.31)$ & $5 / 130(3.85)$ & $>0.05$ \\
\hline Gastrointestinal reactions & $0 / 130(0.00)$ & $3 / 130(2.31)$ & $>0.05$ \\
\hline \multicolumn{4}{|l|}{ Hepatobiliary disorders } \\
\hline Compromised hepatic function & $0 / 130(0.00)$ & $1 / 130(0.77)$ & $>0.05$ \\
\hline \multicolumn{4}{|l|}{ Renal and urinary disorders } \\
\hline Compromised renal function & $0 / 130(0.00)$ & $1 / 130(0.77)$ & $>0.05$ \\
\hline
\end{tabular}
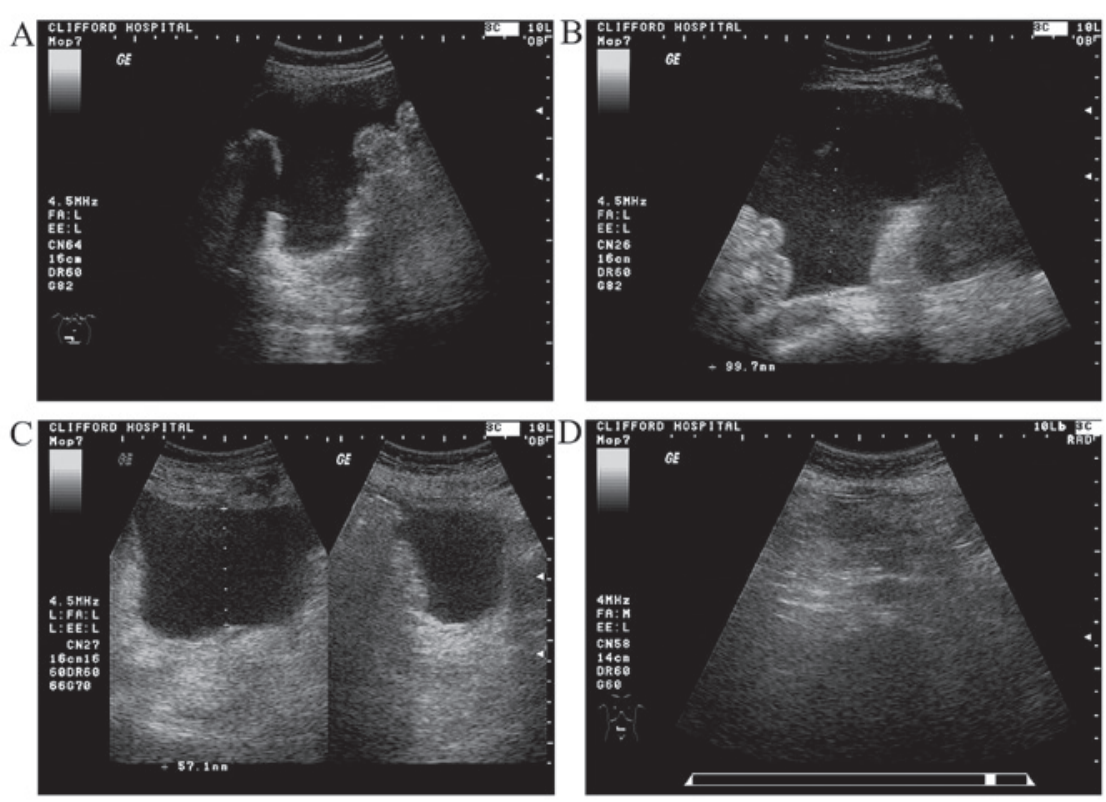

Figure 4. A typical case of a complete response in a 60-year-old postoperative patient with ovarian cancer (oophorohysterectomy in 2007). The patient was diagnosed with multiple extensive metastases in the liver and the abdominal cavity with ascites in 2013; following administration of four courses of IPCI (CDDP + CTX); the ascites resolved. Abdominal distention and edema of the lower limbs was observed in August, 2014, and one course of IPCI was administered, without relief and with associated with adverse reactions such as nausea, vomiting and loss of appetite. (A and B) The patient was admitted to Clifford Hospital on September 12, 2014, with severe malignant ascites (maximum depth, $10.0 \mathrm{~cm}$ ) and ultrasound signs of multiple peritoneal carcinomatosis and a KPS of $60 \%$, with significantly compromised hepatic and renal function on blood tests. mEHT with TCM ('asthenia of both the spleen and kidney' type) treatment was administered according to the study protocol, without adverse reactions. (C) Following completion of the treatment on October 16, 2014, there was relief of the abdominal distention and the edema of the lower limbs; on blood tests, the hepatic and renal function had returned to normal; the ascites was moderate (5.7 cm deep), with a significant reduction in peritoneal carcinomatosis manifestations; the KPS was 90\%. (D) On re-evaluation (November 13, 2014) the hepatic and renal function tests were normal, there was no obvious ascites, no obvious intestinal wall thickening and no characteristic sign of neoplasia; there was also no discomfort and the KPS was $100 \%$. The patient was in complete remission. 
after treatment completion (Fig. 4). This delayed effect of the treatment may be associated with mEHT, which is known to elicit delayed apoptotic reactions $(41,42)$ and to improve immunity $(43,44)$, as well as with the TCM. TCM herbal medicines increase the sensitivity of cancer to mEHT and, vice versa, $\mathrm{mEHT}$ increases the anticancer effect of herbal medicines (9). There are broad prospects for the combination of mEHT with herbal medicines, particularly for patients who are not sensitive to chemotherapy or those who are unable to receive chemotherapy. However, further research is required to study this long-term combined therapeutic effect.

mEHT was used without previous drainage of the ascites, as recommended by the EHY-2000 device user manual. This approach is based on several previous observations of 'as is' mEHT application in cases with tense ascites, with high efficacy and without side effects. This simplified approach significantly reduces time and cost, promotes patients' tolerability to treatment and provides an outstanding advantage in clinical practice.

The main limitations of this trial were the lack of detailed PC characteristics (45) and lack of a survival analysis due to the restricted funding of the trial. Further phase III trials are warranted.

As regards generalizability, the present study suggests that mEHT in combination with TCM is an effective and safe treatment for PCMA. Concordance with previous findings indicates external validity of the present trial's results. In view of efficacy, safety, ease of application and low treatment cost, our results are considered to be worthy of clinical generalization.

In conclusion, the combination of MEHT with TCM may be a preferred treatment option, as it provides better control of PCMA compared with standard IPCI, with less toxicity. Both components of this combination are non-toxic treatments easily tolerated by patients, ensuring a better balance between benefit and harm.

\section{Acknowledgements}

The present trial was funded by the Clifford Hospital.

\section{References}

1. Ayantunde AA and Parsons SL: Pattern and prognostic factors in patients with malignant ascites: A retrospective study. Ann Oncol 18: 945-949, 2007.

2. Sangisetty SL and Miner TJ: Malignant ascites: A review of prognostic factors, pathophysiology and therapeutic measures. World J Gastrointest Surg 4: 87-95, 2012.

3. Roussakow S: Critical analysis of electromagnetic hyperthermia randomized trials: Dubious effect and multiple biases. Conference Papers in Medicine 2013, 2013.

4. Roussakow S: The history of hyperthermia rise and decline. Conference Papers in Medicine 2013, 2013.

5. The American Cancer Society: Hyperthermia to Treat Cancer http://www.cancer.org/treatment/treatmentsandsideeffects/treatmenttypes/hyperthermia. Accessed February 16, 2017.

6. Ma Shenglin: Research progress of thermo-chemo-therapy. Mod Pract Med 16: 256-258, 2004.

7. Szasz A, Szasz N and Szasz O: Oncothermia: Principles and Practices. New York, Springer, NY, 565p, 2011.

8. Andocs G, Renner H, Balogh L, Fonyad L, Jakab C and Szasz A: Strong synergy of heat and modulated electromagnetic field in tumor cell killing. Strahlenther Onkol 185: 120-126, 2009.

9. Pang CLK: Clinical research on integrative treatment of colon carcinoma with oncothermia and clifford TCM immune Booster. Oncothermia J 5: 24-41, 2012.
10. Ling Y: Traditional Chinese medicine in the treatment of symptoms in patients with advanced cancer. Ann Palliat Med 2: $141-152,2013$.

11. Veith I (translator): The Yellow Emperor's Classic of Internal Medicine. (1972). Revised paperback edition. University of California Press, Berkeley, LA, p91, 2002.

12. Zheng Y and Gao F: The recognition of TCM on seroperitoneum of hepatic cirrhosis. J Trad Chinese Med 5: 83, 2008.

13. Zhou D: Oncology of TCM. Guangzhou, Guangdong High Education Publishing House, 90 p, 2007.

14. Chen F: Clinical research of out-of-body high frequency hyperthermia in combination with TCM treating malignant pleural fluid and ascites. J Practical Traditional Chinese Med 27: 686-687, 2011.

15. Zhou L and Zhang S: Clinical observation of adjusted Wu Ling Decoction treating 70 patients with malignant ascites. J Practical Chinese Intern Med 24: 7-711, 2010.

16. Huang X: Observation of Shen Zhu Decoction in combination with endogeny hyperthermia treating 69 patients with malignant ascites. J Practical Chinese Intern Med 20: 388, 2006.

17. Gong S: Observation of the therapeutic effect of microwave hyperthermia in combination with No2.3 Readjusted Decoction treating malignant pleural fluid and ascites. J Changchun University of Traditional Chinese Med 27: 643-644, 2011.

18. Ji Sheng Fang: (8 juan/(Song) Yan Yonghe zhuan). (In Chinese) Taibei, Taiwan shang wu yin shu guan, 149p, 1975.

19. Becker G, Galandi D and Blum HE: Malignant ascites: Systematic review and guideline for treatment. Europ J Cancer 42: 589-597, 2006.

20. Matharu G, Tucker O and Alderson D: Systematic review of intraperitoneal chemotherapy for gastric cancer. Br J Surg 98: 1225-1235, 2011.

21. McRee AJ and O'Neil BH: The role of HIPEC in gastrointestinal malignancies: Controversies and conclusions. Oncology (Williston Park) 29: 523-524,C3, 2015.

22. Verhulst J: Effects of bevacizumab and hyperthermia in a rodent model of hyperthermic intraperitoneal chemotherapy (HIPEC). Int J Hyperthermia 29: 62-70, 2013.

23. Zeamari S, Floot $\mathrm{B}$, van der Vange $\mathrm{N}$ and Stewart FA: Pharmacokinetics and pharmacodynamics of cisplatin after intraoperative hyperthermic intraperitoneal chemoperfusion (HIPEC). Anticancer Res 23: 1643-1648, 2003.

24. Sørensen O, Andersen AM, Kristian A, Giercksky KE and Flatmark K: Impact of hyperthermia on pharmacokinetics of intraperitoneal mitomycin $\mathrm{C}$ in rats investigated by microdialysis. J Surg Oncol 109: 521-526, 2014.

25. Kroon HM and Thompson JF: Isolated limb infusion: A review. J Surg Oncol 100: 169-177, 2009.

26. Di Miceli D, Alfieri S, Caprino P, Menghi R, Quero G, Cina C, Pericoli Ridolfini M and Doglietto GB: Complications related to hyperthermia during hypertermic intraoperative intraperitoneal chemiotherapy (HIPEC) treatment. Do they exist? Eur Rev Med Pharmacol Sci 16: 737-142, 2012.

27. Jafari MD, Halabi WJ, Stamos MJ, Nguyen VQ, Carmichael JC, Mills SD and Pigazzi A: Surgical outcomes of hyperthermic intraperitoneal chemotherapy: Analysis of the american college of surgeons national surgical quality improvement program. JAMA Surg 149: 170-175, 2014.

28. Zhenxia Z, Ziwei L and Ran J: Abdominal indwelling catheter drainage combined with intracavitary perfusion chemotherapy for patients with malignant seroperitoneum. J Huaihai Med 26: 306-528, 2008

29. Chongqi W, Chiping W and Yuying S: The clinical value of 5-FU combined with cisplatin for hyperthermic intraperitoneal chemoinfusion in treating cancerous ascites. Chin J Med Drug Appl 2: 9-10, 2008.

30. de Kock I, Mirhosseini M, Lau F, Thai V, Downing M, Quan H, Lesperance $\mathrm{M}$ and Yang J: Conversion of karnofsky performance status (KPS) and Eastern cooperative oncology group performance status (ECOG) to palliative performance scale (PPS), and the interchangeability of PPS and KPS in prognostic tool. J Palliat Care 29: 163-169, 2013.

31. Ovarian cancer including fallopian tube cancer and primary peritoneal carcinoma. NCCN Clinical Practice Guidelines in Oncology. Ver. 3.2014. https://www.nccn.org/professionals/ physician_gls/f_guidelines.asp. Accessed February 16, 2017.

32. Zhou Daihan, (ed): TCM Oncology. Guangdong High Education Press (ed 1), 2007.

33. Sun Yan: Medical Oncology. Beijing, People's Medical Publishing House, p648-649, 2001. 
34. Hawker GA, Mian S, Kendzerska T and French M: Measures of adult pain: Visual Analog Scale for Pain (VAS Pain), Numeric Rating Scale for Pain (NRS Pain), McGill Pain Questionnaire (MPQ), Short-Form McGill Pain Questionnaire (SF-MPQ), Chronic Pain Grade Scale (CPGS), Short Form-36 Bodily Pain Scale (SF-36 BPS), and Measure of Intermittent and Constant Osteoarthritis Pain (ICOAP). Arthritis Care Res (Hoboken) 63 (Suppl 11): S240-S252, 2011

35. Common Terminology Criteria for Adverse Events (CTCAE) (v4.03: June 14, 2010) U.S. Department of Health and Human Services, National Institutes of Health, National Cancer Institute.

36. Moher D, Hopewell S, Schulz KF, Montori V, Gøtzsche PC Devereaux PJ, Elbourne D, Egger M and Altman DG; CONSORT: CONSORT 2010 Explanation and Elaboration: Updated guidelines for reporting parallel group randomised trials. Int J Surg 10: $28-55,2012$

37. Li Z, Zhang L and Li L: Treatment of malignant ascites with hyperthermic perfusion chemotherapy and high-frequency hyperthermia. Med J West China 22: 517-521, 2010.

38. Yu X, Li X, Zhou J, et al: The clinical study of intraperitoneal chemotherapy combined with whole body hyperthermia by using microwave on abdomen for treating malignant peritoneal effusion. J Clin Intern Med 24: 253-255, 2007.

39. Yin J, Dai P and Xie Z: Clinical study of chemotherapeutic hyperthermia intraperitoneal perfusion combined with high frequency hyperthermia for the treatment of malignant ascites. Med J Wuhan University 28: 248-250, 2007.

40. Wang H, Liu P, Wang Y, et al: Effects observation of treating malignant ascites with in vitro radiofrequency thermotherapy combined intraperitoneal perfusion chemotherapy. China Clinicians 43: 31-32, 2015.

41. Meggyeshazi N, Andocs G, Balogh L, Balla P, Kiszner G, Teleki I, Jeney A and Krenacs T: DNA fragmentation and caspase-independent programmed cell death by modulated electrohyperthermia. Strahlenther Onkol 190: 815-822, 2014.
42. Andocs G, Meggyeshazi N, Balogh L, Spisak S, Maros ME, Balla P, Kiszner G, Teleki I, Kovago C and Krenacs T: Upregulation of heat shock proteins and the promotion of damage-associated molecular pattern signals in a colorectal cancer model by modulated electrohyperthermia. Cell Stress Chaperones 20: 37-46, 2015.

43. Tsang YW, Huang CC, Yang KL, Chi MS, Chiang HC, Wang YS, Andocs G, Szasz A, Li WT and Chi KH: Improving immunological tumor microenvironment using electro-hyperthermia followed by dendritic cell immunotherapy. BMC Cancer 15: 708, 2015.

44. Akutsu Y, Tamura Y, Murakami K, et al: Can modulated electro-hyperthermia (mEHT) elicit immune reaction? -From basic and clinical research. Therm Med 30: 62 (WS1-WS1-3), 2014.

45. Sugarbaker H: Technical Handbook for the Integration of Cytoreductive Surgery and Perioperative Intraperitoneal Chemotherapy into the Surgical Management of Gastrointestinal and Gynecologic Malignancy. 4th edition. Ludann Company, Grand Rapids, MI, p67, 2005. 